\title{
Fractional Order System and Hळ: A Literature Survey
}

\author{
Saurabh Deshmukh ${ }^{1}$, Sagar Darade ${ }^{2,}$ Bharat Gaikwad ${ }^{3}$, Sagar Darade $^{4}$ \\ 1,2,3,4 (Department of Electronics and Telecommunication, Saraswati college of Engineering ,Mumbai University
} , India.)

\begin{abstract}
We present here a detailed survey of H-infinity $H_{\infty}$ norm computation for a commensurate Fractional Order System (FOS). First, Ho norm definition is given for FOS and then Hamiltonian matrix of a FOS is computed. One method based on this Hamiltonian matrix is then proposed to compute the FOS Ho norm which is based on a dichotomy algorithm.
\end{abstract}

Keywords: FOC-fractional order system; $\boldsymbol{H} \infty$-h-infinity commensurate-corresponding in size or degree; in proportion.Hamiltonian matrix-In mathematics, a Hamiltonian matrix is a 2n-by-2n matrix A such that JA is symmetric, where $J$ is the skew-symmetric matrix.Riemann-Liouville integral-In mathematics, the RiemannLiouville integral associates with a real function $f: \boldsymbol{R} \rightarrow \boldsymbol{R}$ another function $I^{\alpha} f$ of the same kind for each value of the parameter $\alpha>0$.

\section{Introduction}

Students of mathematics, sciences, and engineering encounter the differential operators $d / d x, d 2 / d x 2$, etc., but probably few of them ponder over whether it is necessary for the order of differentiation to be an integer. Why not be a rational, fractional, irrational, or even a complex number? At the verybeginning of integral and differential calculus, in a letter to L'H'opital in 1695, Leibniz himself raised the question: "Can the meaning of derivatives with integer order be generalized to derivatives with non-integer orders?' $L$ 'H'opital was somewhat curious about that question and replied by another question to Leibniz: "What if the order will be 1/2?" Leibniz in a letter dated September 30, 1695 replied: "It will lead to a paradox, from which one day useful consequences will be drawn." The question raised by Leibnizfor a non-integer-order derivative was an ongoing topic for more than 300 years, and now it is known as fractional calculus, a generalization of ordinary differentiation and integration to arbitrary (non-integer) order.

Before introducing fractional calculus and its applications to control in this book, it is important to remark that "fractional," or "fractional-order," are improperly used words. A more accurate term should be "non-integerorder," since the order itself can be irrational as well. However, a tremendous amountof work in the literature use "fractional" more generally to refer to the same. For this reason, we are using the term "fractional" in this paper

\section{Why Fractional Order?}

It is usual in undergraduate courses of feedback control to introduce the basic control actions and their effects in the controlled system behavior, in the frequency domain. So, we know that these actions are proportional, derivative, and integral, and their main effects over the controlled system behavior are -

1. To increase the speed of the response, and to decrease the steady-state error and relative stability, for proportional action; to increase the relative stability and the sensitivity to noise,for derivative action; To eliminate the steady-state error and to decrease the relative stability for integral action. The positive effects of the derivative action (increased relative stability) can be observed in the frequency domain by the $\pi / 2$ phase lead introduced, and the negative ones (increased sensitivity to high-frequency noise) by the increasing gain with slope of $20 \mathrm{~dB} / \mathrm{dec}$. For the integral action, the positiveeffects (elimination of steady-state errors) can be deduced by the infinite gain at zero frequency, and the negative ones (decreased relative stability) by the $\pi / 2$ phase lag introduced.

2. Considering this, it is quite natural to conclude that by introducing more general control actions of the form sn, $1 / \mathrm{sn}, \mathrm{n} \in \mathrm{R}+$, we could achieve more satisfactory compromises between positive and negative effects, and combining the actions we could develop more powerful and flexible design methods to satisfy the controlled system specifications.

3..Let us turn now our attention from feedback control to systems modeling. Researchers in electrochemistry, biological systems, material science, viscoelasticity, and other fields in which electrochemical, mass transport, diffusion, or other memory phenomena appear [2-4], usually perform frequencydomain experiments in order to obtain equivalent electrical circuits reflecting the dynamic behavior of the systems under study. 
4.It is quite usual in these fields to find behaviors that are far from the expected ones for common lumped elements such as resistors, inductors, and capacitors, and to definefor operational purposes special impedances such as Warburg impedances, constant phase elements (CPEs), and others.

5.All these special impedances have in common a frequency domain behavior of the form $\mathrm{k} /(\mathrm{j} \omega) \mathrm{n}, \mathrm{n} \in \mathrm{R}$, and so, in the Laplace domain these elements should be modeled by $\mathrm{k} / \mathrm{sn}, \mathrm{n} \in \mathrm{R}+$. These operators in the frequency and Laplace domains give rise to the corresponding operators in the time domain. In what follows, by using standard definitions of repeated integrals and derivatives, we will try to show that these operators that arise in a quite natural way in the frequency domain, lead us to the definition of differential and integral operators of arbitrary order, the fundamental operators of the fractional calculus.

\section{Indentations and Equations}

the function

Let us assume for the time being zero initial conditions. If we define $F(s)$ as the Laplace transform of $\mathrm{f}(\mathrm{t}), \mathrm{F}(\mathrm{s}) \equiv \mathrm{L}[\mathrm{f}(\mathrm{t})]$, in the equation

$\underline{1} \mathrm{~F}(\mathrm{~s})$, sn

\subsection{Why Fractional Order?}

1.2 We can recognize the Laplace domain equivalent for the $n$-fold integral of the function $\mathrm{f}(\mathrm{t})$. Consider an antiderivative or primitive of the function

$\mathrm{f}(\mathrm{t}), \mathrm{D}^{-1} \mathrm{f}(\mathrm{t})$, then

$$
\mathrm{D}^{-1} \mathrm{f}(\mathrm{t})=\int_{0}^{t} f(x) d x
$$

Now let us perform the repeated applications of the operator. For example,

$\mathrm{D}^{-2} \mathrm{f}(\mathrm{t})=\int_{0}^{t} \int_{0}^{x} f(y) d y d x$

Equation 1.3 can be considered as a double integral, and taking into account the $x-y$ plane over which it is integrated (see Figure 1.1), we can reverse the sequence of integrations by doing the proper changes in their limits. So, we obtain

$\mathrm{D}^{-2} \mathrm{f}(\mathrm{t})=\int_{0}^{t} \int_{y}^{t} f(y) d x d y$

\section{Figures and Tables}

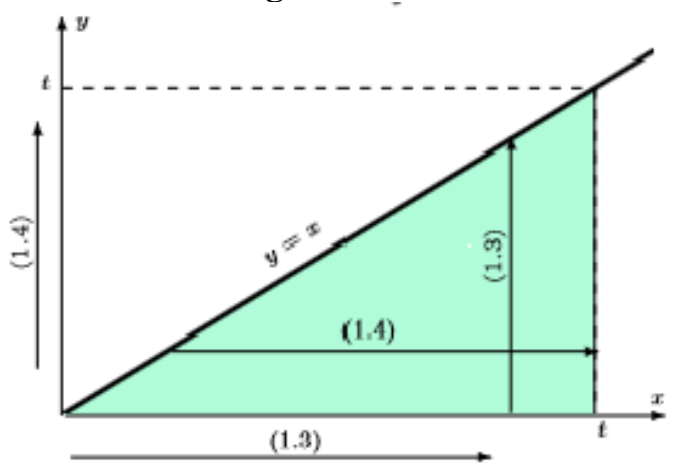

- LTI Commensurate Fractional Order Systems

In this paper we consider LTI commensurate FOS admitting a pseudo state space representation of the form

$$
\begin{array}{ll}
\mathrm{D} v_{\mathrm{x}}(\mathrm{t})= & \mathrm{Ax}(\mathrm{t})+\mathrm{Bu}(\mathrm{t}) ; \\
\mathrm{y}(\mathrm{t})= & \mathrm{Cx}(\mathrm{t})+\mathrm{Du}(\mathrm{t}) \ldots
\end{array}
$$

where $\mathrm{x}(\mathrm{t}) \varepsilon \mathrm{R}^{\mathrm{n}}$ is the pseudo state vector, $\mathrm{u}(\mathrm{t}) \varepsilon \mathrm{R}^{\mathrm{m}}$ is the input vector, $\mathrm{y}(\mathrm{t}) \varepsilon \mathrm{R}^{\mathrm{p}}$ is the output vector, $v$ is the fractional order of the system and A, B, C and D are constant matrices.Dv is the fractional differentiation operator of order $v$ (presented results are valid whatever definition used: Riemann-Liouville, Caputo [3].Transfer matrix is $\mathrm{G}(\mathrm{s})=\mathrm{C}\left(\mathrm{s}^{\mathrm{V}} \mathrm{I}-\mathrm{A}\right)^{-1} \mathrm{~B}+\mathrm{D}$ andimpulse response matrix is,

$$
g(t)=L^{-1} G(S) \text {. }
$$


- $\quad$ Stability of commensurate fractional order systems

Definition 1 (Matignon, 1996 [2] ): A linear FOS defined by its impulse response $\mathrm{g}$ is Bounded-Input BoundedOutput (BIBO) stable iff $\forall v \varepsilon \mathrm{L} \propto\left(\mathrm{R}^{+}, \mathrm{R}^{\mathrm{m}}\right), \mathrm{y}=\mathrm{g} \star v \varepsilon \mathrm{L} \propto\left(\mathrm{R}^{+}, \mathrm{R}^{\mathrm{m}}\right.$ Theorem 1 (Matignon, 1996 [2]): System, with minimal triplet $(\mathrm{A}, \mathrm{B}, \mathrm{C})$ and $0<v<1$, is BIBO stable iff $|\arg (\operatorname{eig}(\mathrm{A}))|>v \frac{\pi}{2}$

This result remains valid when $1<v<2$ as proved in [?] Stability domain is thus defined as follows:

$\mathrm{Ds}=\left\{\mathrm{z \varepsilon C}:|\arg (\operatorname{eig}(\mathrm{A}))|>v \frac{\pi}{2}\right\}$,

\section{- Ho Norm Of A Commensurate Fractional Order System}

As for LTI IOS [5], let us define the Ho norm of stable FOS from its transfer function G(s) as follows: Definition $2: \mathrm{H} \infty$ norm of stable FOS system is:

$\|\mathrm{G}(\mathrm{s})\| \infty=\max \sigma(\mathrm{G}(\mathrm{j} \omega))$,

Worst case frequency gain is thus given by $\mathrm{H} \infty$ norm of FOS:

$\|\mathrm{G}(\mathrm{s})\| \infty=\max \max \|\mathrm{y}(\mathrm{j} \omega)\| 2$

$\omega \varepsilon R \quad u(j \omega) \neq 0 \quad\|u(j \omega)\| 2$

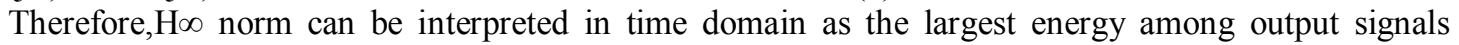
resulting from all inputs of unit energy. Consequently, $\mathrm{H}_{\infty}$ norm physical interpretation, in frequency and time domains, is the same for

FOS as for IOS.

In time domain, equation (5) can be written as:

$$
\|\mathrm{G}(\mathrm{s})\| \infty=\max \quad \max \quad\|\mathrm{y}(\mathrm{j} \omega)\| 2
$$

$\mathrm{u}(\mathrm{t}) \quad \mathrm{u}(\mathrm{j} \omega) \neq 0 \quad\|\mathrm{u}(\mathrm{j} \omega)\| 2$

Therefore, $\mathrm{H} \infty$ norm can be interpreted in time domain as the largest energy among output signals resulting from all inputs of unit energy. Consequently, Hळo norm physical interpretation, in frequency and time domains, is the same for FOS as for IOS.

\section{Hœ NORM COMPUTATION}

A)FOS Hamiltonian matrix

The Ho norm of IOS is usually computed numerically from a state-space realization as the smallest value of $\gamma$ such that the Hamiltonian matrix $\mathrm{H} \gamma$ has no eigen value on the imaginary axis [6].

This section develops a similar result for FOS. Ho norm upper bound inequality: Definition 2 imply that $\mathrm{H} \infty$ norm of FOS is less than $\gamma$ iff:

$$
\omega \varepsilon \mathrm{R} \max _{\mathrm{i}=1 \ldots \min (\mathrm{m}, \mathrm{p})} \sqrt{\lambda \mathrm{i}(\mathrm{G}(\mathrm{j} \omega)) *(\mathrm{G}(\mathrm{j} \omega))}<\gamma,
$$

The squared power of last inequality is:

$\omega \varepsilon \mathrm{R} \max \quad \lambda_{\mathrm{i}}(\mathrm{G}(\mathrm{j} \omega)) *(\mathrm{G}(\mathrm{j} \omega))<\gamma^{2}$,

Using eigenvalues properties, relation $(8)$ becomes:

$$
\omega \varepsilon R \quad \max \quad \lambda_{i}\left(\gamma^{2} \mathrm{I}-\mathrm{G}(\mathrm{j} \omega)\right) * \mathrm{G}(\mathrm{j} \omega)>0,
$$

which is equivalent to the $\mathrm{H} \infty$ upper bound inequality:

$$
\omega \varepsilon \mathrm{R}\left(\gamma^{2} \mathrm{I}-\mathrm{G}(\mathrm{j} \omega)\right) * \mathrm{G}(\mathrm{j} \omega)>0,
$$

Relation (11) is an infinite dimension inequality since it depends on $\omega \varepsilon R$. The next two subsections show how to make this relation independent of $\omega$. The first step is to build a pseudo state-space representation of

$$
\Phi(\mathrm{s})=\gamma^{2} \mathrm{I}-\mathrm{G}(\mathrm{s}) * \mathrm{G}(\mathrm{s})
$$

B) Pseudo state-space representation of $\phi(s)$ :

Pseudo state-space representation of $\phi(s)$ is:

$$
\left(\begin{array}{c}
D v^{\sim}(t)=A^{\sim}(t)+{ }^{\sim} B^{\sim}(t) \\
\sim(t)=C^{\sim}(t)+{ }^{\sim} D^{\sim}(t)
\end{array}\right)
$$

\section{C) Hamiltonian matrix theorem:}

Theorem 2: Let $\gamma>\sigma(\mathrm{D})$ be a positive real number [7].

Then $\|\mathrm{G}(\mathrm{s})\| \infty<\gamma$ iff fractional order Hamiltonian matrix $\mathrm{H} \gamma$ is equal to equation(12):

$$
\begin{array}{cc}
\left(\begin{array}{cc}
A+B R D^{\wedge} T C & B R B^{\wedge} T \\
\exp { }^{\wedge} \mathrm{vj} \Pi \mathrm{C}^{\wedge} \mathrm{T}\left(1+\mathrm{DRD}^{\wedge} \mathrm{T}\right) \mathrm{C} & \exp ^{\wedge} \mathrm{vj} \Pi\left(\mathrm{A}^{\wedge} \mathrm{T}+\mathrm{C}^{\wedge} \mathrm{T} \mathrm{DRB}^{\wedge} \mathrm{T}\right)
\end{array}\right) \\
\text { where } \mathrm{R}=\left(\gamma^{2} \mathrm{I}-\mathrm{D}^{\mathrm{T}} \mathrm{D}\right)^{-1} & \text { has no eigenvalue in set }
\end{array}
$$




$$
\mathrm{C}_{\mathrm{vo}}=\mathrm{j} \omega^{\mathrm{v}}=\omega^{\mathrm{v}} \exp ^{\mathrm{v} \mathrm{j}_{\frac{\pi}{2}}^{\mathrm{T}}}, \omega \varepsilon \mathrm{R}
$$

\section{D) $\gamma$-iteration}

This method for $\mathrm{H} \infty$ norm computation is a dichotomous optimization process directly derived from theorem 2 [8]. The following algorithm shows $\gamma$-iteration for a FOS:

1. Choose $\left[\gamma_{\min }, \gamma_{\max }\right]$ such that $\gamma_{\min }>\sigma^{-}(\mathrm{D})$

2. For $\gamma=\left(\gamma_{\min }+\gamma_{\max }\right) / 2$, determine $\mathrm{H} \gamma$ eigenvalues.

3. If the eigenvalues are not in set $\mathrm{Cv} 0, \gamma$ is reduced by taking a new interval $\left[\gamma_{\min }, \gamma\right]$.

4.If the eigenvalues are in set $\mathrm{C} v 0, \gamma$ is increased by taking a new interval $\left[\gamma_{\min }, \gamma\right]$.3. Step 2 is repeated until $\gamma$ gives a satisfactory approximation of $\mathrm{H} \infty \mathrm{n}$ norm.

\section{Conclusion}

THE MAJOR CONTRIBUTIONS OF THIS PAPER ARE:-

- HAMILTONIAN MATRIX DEFINITION FOR A FOS ;

- ONE METHOD BASED ON THE T-ITERATION.

• OUR FUTURE WORKS AIM NOW TO FIND A LINEARIZING CHANGE OF VARIABLE IN THE PROPOSED

HAMILTONIAN MATRIX DEFINITION THAT WILL PERMIT TO FIND DIRECTLY, WITHOUT AN ITERATIVE PROCESS, THE FOS Hळ NORM.

\section{References}

[1] C. andJ.R Partington, “Analysis of fractional delay systems,” Automatica, vol. 38(7), pp. 1133-1138, 2006.

[2] D.Matigon, "Stability result on fractional differential equations with applications to control processing," vol. 2, pp. 963-968, Computational Engineering in Systems Application, July 2003.

[3] C.Farges and M.Moze, "Pseudo-state feedback stabilization of commensurate Fractional order systems," Automatica, vol. 46(10), pp. $1730-1734,2010$.

[4] M.Moze, An Introduction to fractional differential equations. PhD thesis, ENSEIRB,Bordeaux, 2008

[5] S. Skogestad, Multivariable Feedback Control. John Wiley and Sons, 2005.

[6] K. j.Doyle, Robust and Optimal Control. Prentice Hall, 1998

[7] S.Boyd and L.Vandenberghe, Convex Optimization. 2004.

[8] S.Byod and E.Feron, Linear Matrix Inequalities in system and Control Theory. Philadelphia, 2004. 